\title{
HYDROGEN: THERAPEUTIC POTENTIAL IN WELLNESS AND MEDICINE
}

\author{
M. Lemaire', F. Barbier ${ }^{2}$
}

\begin{abstract}
Persistent oxidative stress plays an important role in a variety of pathologies, and the search for an effective and well tolerated antioxidant agent continues. Molecular hydrogen acts as a therapeutic antioxidant by selectively reducing cytotoxic oxygen radicals. The non-specific mechanism of hydrogen as a therapeutic antioxidant gives it broad therapeutic potential across a wide range of medical applications, as has been shown by a substantial volume of preclinical data, as well as a growing body of clinical evidence. This review provides an overview of the therapeutic potential of hydrogen, in ageing and wellness applications as well as medical applications, including acute ischemia/reperfusion injury, inflammation and ulceration, metabolic disorders, neurodegenerative disorders, and cancer (anti-cancer effects, radiation toxicities, and side effects of cisplatin) with an emphasis on clinical data. Overall, this review shows that hydrogen is an effective antioxidant, anti-inflammatory and cytoprotective agent.
\end{abstract}

Key words: Hydrogen, ageing, wellness, inflammation, antioxidant.

\section{Introduction}

Hydrogen, a single proton and a lone electron, is the lightest and most abundant element in the universe. On Earth, hydrogen generally exists in compounds with other elements, and is not found by itself. The most common sources of hydrogen are hydrocarbons, water and carbohydrates from which molecular hydrogen can be produced (1).

With more than 50 million tons produced globally each year, hydrogen is one of the oldest known molecules that is used extensively by many industries. Most of its uses are based on its physical properties, such as its very low molecular weight $(2.0159 \mathrm{~g} / \mathrm{mol} \mathrm{H} 2)$ and density $\left(0.084 \mathrm{~g} / 1\right.$ at $20^{\circ} \mathrm{C}$ and $\left.1 \mathrm{~atm}\right)$, and its chemical properties such as its reactivity, forming compounds or preventing oxidation of metals due to its reducing properties (1). Hydrogen has the potential to become an important future energy solution due its high calorific value and its environmentally friendly properties, generating only water vapor when used in fuel cells to produce electricity. The benefits of molecular hydrogen in therapeutic applications have been discovered relatively recently, and remain to be completely elucidated. Many consider

1. Air Liquide Sante International, Paris Saclay Research Center, Jouy-en-Josas, France, 2. Air Liquide R\&D, Paris Saclay Research Center, Jouy-en-Josas, France.

Corresponding Author: Marc Lemaire, Air Liquide Sante International, Paris Saclay Research Center, 1, route de la porte des loges, Les Loges-en-Josas, 78354, Jouyen-Josas, France. Tel: +33139076212; Fax:+33139076199; email: Marc.lemaire@ airliquide.com

Received June 24, 2016

Accepted for publication June 27, 2016 hydrogen to have healing properties, with its role as the fundamental antioxidant for every cell. Reactive oxygen species (ROS) are generated in the bodies of all aerobic organisms as by-products of energy metabolism via oxidative phosphorylation (2). Excess production of ROS or impaired endogenous antioxidant capacity result in a build-up of ROS, known as oxidative stress (2). It is widely accepted that persistent oxidative stress plays an important role in a variety of pathologies, including lifestyle-related diseases such as atherosclerosis and diabetes mellitus, hypertension, chronic inflammation, neurodegenerative diseases, cancer, decreased vascular function and the ageing process (2-7). However, the majority of prospective controlled clinical trials of antioxidant therapy to prevent oxidative stress-related diseases, mostly cardiovascular indications, have failed (8). Therefore, the search for an effective and well tolerated antioxidant agent continues.

Hydrogen is an odorless, tasteless gas that has demonstrated many biological effects against oxidative stress in almost all organs $(9,10)$. Molecular hydrogen acts as a therapeutic antioxidant by selectively reducing cytotoxic oxygen radicals (11). Hydrogen has a number of characteristics that suggest it might be an ideal antioxidant agent for use in humans. These include its ability to neutralize hydroxyl radicals $(\bullet \mathrm{OH})$ in living cells (including penetration into organelles such as mitochondria) and to cross the blood-brain barrier, as well as its stability at room temperature and low solubility in water $\left(1.9 \mathrm{~mL} \mathrm{H} 2 / 100 \mathrm{~mL} \mathrm{H} 2 \mathrm{O}\right.$ at $20^{\circ} \mathrm{C}$ and $1 \mathrm{~atm}$, Table 1$)$ and a favorable tolerability profile $(2,10$, 
Table 1

Solubility of hydrogen in water

\begin{tabular}{|c|c|c|c|c|}
\hline Units & $\underset{\mathrm{H}_{2} \mathrm{O}_{\text {(liquid) }}}{\mathrm{mg} \mathrm{\textrm {H } _ { 2 }}}$ & $\begin{array}{c}\mathrm{ml} \mathrm{H}_{2 \text { (gas) }} / 100 \mathrm{ml} \\
\mathrm{H}_{2} \mathrm{O}_{\text {(liquid) }}\end{array}$ & $\begin{array}{l}\text { ppmw (part per million } \\
\text { weight) }\end{array}$ & $\begin{array}{c}\mathrm{mM} \text { (millimolar } \\
\text { concentration) } \\
\mathrm{mmol} \mathrm{H}_{2 \text { (gas) }} / 100 \mathrm{ml} \\
\mathrm{H}_{2} \mathrm{O}_{\text {(liquid) }} \\
\end{array}$ \\
\hline $\mathrm{H}_{2}$ solubility in water ${ }^{*}\left(20^{\circ} \mathrm{C}, 1 \mathrm{~atm}\right)$ & 0.16 & 1.9 & 1.6 & 0.08 \\
\hline $\begin{array}{l}\mathrm{H}_{2} \text { enriched-water (drink, injection...) } \\
\text { used in literature }\end{array}$ & 1 & & 10 & 0.5 \\
\hline
\end{tabular}

*From Young C.L., 1981 (18).

Table 2

Summary of the therapeutic advantages of hydrogen as an antioxidant

\begin{tabular}{|c|c|}
\hline Physical and Chemical Properties & Molecular Hydrogen Features \\
\hline Low molecular weight & $\begin{array}{l}\text { Rapid and easy diffusion across cellular membranes to reach key intracellular } \\
\text { structures (e.g. mitochondria) }\end{array}$ \\
\hline Small size, high diffusivity & Crosses the blood-brain barrier \\
\hline Strong covalent bond between hydrogen atoms & Stability at room and body temperature \\
\hline $\mathrm{H} 2$ molecule is non-polar & Slightly soluble in water at atmospheric pressure \\
\hline Reducing agent - neutralizes toxic free radicals such as $\bullet \mathrm{OH}$ & Selective antioxidant effects \\
\hline $\begin{array}{l}\text { Selective reducing agent - does not affect important ROS } \\
\text { such as } \bullet \mathrm{O} 2 \text { - and } \mathrm{H} 2 \mathrm{O} 2\end{array}$ & No effect on redox homeostasis \\
\hline \multirow[t]{2}{*}{ Reaction with the hydroxyl radical } & Multiple mechanisms of action: \\
\hline & - antioxidant \\
\hline $\begin{array}{l}\text { Reaction with the hydroxyl radical; inhibition of } \\
\text { lipopolysaccharide/interferon } \gamma \text {-induced nitric oxide } \\
\text { production }\end{array}$ & - anti-inflammatory \\
\hline Possibly via reducing/ eliminating $\bullet \mathrm{OH}$ and $\mathrm{ONOO}-$ & - anti-apoptotic \\
\hline Gas at room temperature & Can be given via a variety of routes including oral, injection and inhalation \\
\hline Antioxidant, anti-inflammatory agent, anti-apoptotic agent & Application across a wide range of wellness and medical applications \\
\hline Endogenous gas - no cytotoxicity & Well tolerated \\
\hline $\begin{array}{l}\text { Dissolution concentration in water (less than } 2 \% \mathrm{v} / \mathrm{v} \text { ) } \\
\text { is well below the lower flammability limit of molecular } \\
\text { hydrogen }(4 \% \mathrm{v} / \mathrm{v}) \text { in air needed for reaction with oxygen }\end{array}$ & Biologically safe and inert with the body \\
\hline
\end{tabular}

$\bullet \mathrm{OH}=$ hydroxyl radical; $\mathrm{ROS}=$ reactive oxygen species; $\bullet \mathrm{O} 2$ - = superoxide; $\mathrm{H} 2 \mathrm{O} 2$ : hydrogen peroxide; ONOO-: peroxynitrite

12). The advantageous physical and chemical properties of hydrogen, in relation to its therapeutic benefits are summarized in Table 2. Therefore, hydrogen may be potentially useful in the many human diseases known to be associated with oxidative stress, and may have an important role in the prevention of ageing and in many wellness applications.

In addition to its role as an anti-oxidant, there is also evidence to suggest that molecular hydrogen has anti-inflammatory properties, via its reaction with the hydroxyl radical $(\bullet \mathrm{OH})$, which is generated during inflammation $(13,14)$. There is also evidence to suggest that hydrogen acts more directly on inflammation, via the inhibition of lipopolysaccharide/interferon $\gamma$-induced nitric oxide production, via modulation of signal transduction in macrophages (15). Hydrogen may therefore also be therapeutically useful in the many inflammatory human diseases. Moreover, hydrogen has also been shown to prevent apoptosis, possibly via its ability to reduce or eliminate the hydroxyl radical $(\bullet \mathrm{OH})$ and peroxynitrite (ONOO-) (16).

Importantly, there are a wide variety of options for the administration of hydrogen, including inhalation as a medical gas, drinking of hydrogen-enriched water (dissolved hydrogen gas, created by exposing water to magnesium, by dissolving electrolyzed hydrogen into water, or by using high pressure to dissolve molecular hydrogen in water $(16,17))$, taking a hydrogen bath, injection of hydrogen-enriched saline and use of hydrogen-enriched saline eye drops (12).

It is important to note that due to the low solubility of hydrogen in water (18), the resulting concentration 
of hydrogen less than $2 \% \mathrm{v} / \mathrm{v}$ is much lower than the concentration of hydrogen $(4 \% \mathrm{v} / \mathrm{v})$ needed to react with oxygen (1). As a result, hydrogen can be considered as safe and inert within the human body.

This review provides an overview of the therapeutic potential of hydrogen. Ageing and wellness applications are discussed first, followed by a variety of medical applications.

\section{Ageing}

Free radicals and reactive oxygen species (ROS) produced by aerobic metabolism play important roles in signal transduction, but also cause damage in biological systems (19). These free radicals and ROS are known to play a role in many human diseases and to be involved in premature ageing (Sun X, 2015).

The search for a simple method to delay or halt the ageing process has been going on for centuries based on efficient anti-oxidant products. Growing evidence of the ability of hydrogen, either as a gas or solution, to scavenge and selectively inactivate ROS suggest that it could exert protective effects (20). Bathing every day for 3 months in waters containing molecular hydrogen $(\mathrm{N}=18)$ has been shown to decrease the oxidation-reduction potential (ORP) and increased the elasticity of human skin $(21,22)$.

The ORP of the skin is increased by sun exposure and by ageing (23), suggesting that a reduction in the ORP might have anti-ageing effects.

\section{Wellness}

Hydrogen has been shown to be alkaline in water (24, 25). Hydrogen-rich alkaline water obtained by adding $\mathrm{H} 2$ to electrolyzed water reduces the $\mathrm{pH}$, but the hydrogen itself that can be expected to be of benefit (26). This has important implications for the wellness industry leading to the development of a beverage or food supplement that is able to take advantage of this hydrogen-associated alkalinity.

\section{Exercise performance and sports injury}

Exercise-induced production of free radicals is now a well-known phenomenon (27). The increased oxidative stress associated with exercise has the potential to diminish the overall beneficial effects that exercise has on wellbeing.

An open-label pilot study in 19 young, healthy men showed that daily intake of $2 \mathrm{~L}$ of hydrogen-enriched water (1.1 $\mathrm{mM}$ of dissolved hydrogen) for 7 days increased fasting and post-exercise blood $\mathrm{pH}$, reflecting a reduction in exercise-associated acidosis, with no adverse effects (28). Athletes receiving $1 \mathrm{~L}$ of hydrogen water daily showed a significant decrease in the rates of perceived exertion during exercise testing (29), and an attenuation of increases in blood lactate levels and prevention of the post-exercise decrease in peak torque, an indicator of muscle fatigue (30).

To investigate the effects of hydrogen in the management of sports injuries, 12 professional athletes presenting within the first 24 hours after a muscle and tendon strain were randomized in a double-blind study to receive standard care alone (control) or in combination with oral hydrogen tables ( $2 \mathrm{~g} /$ day) and topical hydrogen-enriched packs (6 times per day for 20 minutes). Follow-up after 7 days showed that the addition of hydrogen significantly reduced plasma viscosity $(p=0.002)$ and limb circumference $(p=0.04)$. These results indicate that the addition of oral and topical molecular hydrogen to traditional first-line treatment is effective in the management of musculotendinous injuries (31).

\section{Endothelial function}

The results of a study in healthy volunteers suggest that hydrogen might contribute to wellness via modulation of vascular vasomotor function secondary to suppression of subclinical vascular inflammation (32). Thirty minutes after drinking $530 \mathrm{~mL}$ of hydrogenenriched water (each $500 \mathrm{~mL}$ containing $3.5 \mathrm{mg}$ of $\mathrm{H} 2$ ), healthy volunteers showed significantly improved flowmediated dilation in the brachial artery compared with subjects who drank placebo water $(\mathrm{p}=0.0221)$. Potential mechanisms proposed for this effect were neutralization of ROS to preserve NO bioavailability, and suppression of inflammatory pre-atherosclerotic events.

\section{Medical applications}

\section{Major syndromes}

\section{Acute ischemia/reperfusion injury}

\section{Stroke and myocardial infarction}

For both stroke and myocardial infarction the successful treatment strategies to restore blood flow to ischemic tissue are associated with reperfusion injury. One mechanism thought to contribute to this negative outcome is the generation of ROS (33).

In an animal model, inhalation of $2 \%$ hydrogen prior to reperfusion has been shown to attenuate ischemia/ reperfusion injury (IRI) occurring during coronary artery reperfusion, with a significant reduction in infarct size, (41.6\% vs $21.2 \%: \mathrm{p}<0.05)$ (34). Hydrogen-rich saline (0.6 $\mathrm{mM}$ ) reduced the apoptosis IRI, (TUNEL-positive cells: $15 \%$ vs $24 \%$ : p $<0.05)$, decreased infarct size $(9.8 \%$ vs $32.1 \%: \mathrm{p}<0.01)$ and improved heart function parameters 24 hours after reperfusion (35). Hydrogen-enriched saline 
$(0.6 \mathrm{mM})$ has also been shown to decrease infarct size $(\mathrm{p}<0.05)$ as well as both oxidative stress and inflammation (36). In mice, inhalation of hydrogen $(2 \%)$, alone and in combination with nitric oxide (NO), reduced infarct size after IRI. The hydrogen/NO combination was also associated with preservation of cardiac function after IRI (37). These findings suggest that patients may benefit from hydrogen inhalation as an add-on to percutaneous coronary intervention procedures.

ROS are generated during cerebral IRI and contribute significantly to brain injury. Inhalation of hydrogen at $2 \%$ to $4 \%$ during reperfusion decreased infarct volume in rats after middle cerebral artery occlusion and reperfusion $(p<0.001)$; inhalation of hydrogen during ischemia had no effect on infarct size in this model (11), hence to have an effect, hydrogen must be used following reperfusion, as is the case for all organ protective molecules.

In experimental hepatic IRI, inhalation of hydrogen ( $1 \%$ to $4 \%)$ and intraperitoneal injection of hydrogenenriched saline $(2.5,5$ or $10 \mathrm{~mL} / \mathrm{kg})$ have both been associated with reductions in the severity of liver injury, improved liver function and decreases in markers of lipid peroxidation and inflammation $(38,39)$. Similar findings have been reported after treatment with hydrogen-rich saline $(>0.6 \mathrm{mM})$ in rats subjected to renal IRI (40).

In a clinical setting, the addition of intravenous hydrogen-rich saline $(>250 \mu \mathrm{mol} / \mathrm{L})$ to treatment with the hydroxyl radical scavenger/neuroprotective agent edaravone in acute brainstem infarction patients was associated with incremental improvements in magnetic resonance imaging (MRI) findings compared with the natural course: relative diffusion-weighted images (rDWIs), regional apparent diffusion coefficients (rADCs), and pseudo-normalization time of rDWI and rADC were all improved with the combined infusion of hydrogen and edaravone (41).

\section{Cardiac arrest and hemorrhagic shock}

Intraperitoneal injection of hydrogen (10 or $20 \mathrm{~mL} /$ $\mathrm{kg}$ ) reduced neuronal injury and inhibited neuronal apoptosis in a rabbit model of cardiac arrest; these effects appeared to occur via reductions in oxidative stress indicators and enhanced antioxidant enzyme activity (42). Similar beneficial effects have been observed with inhalation of hydrogen $(2 \% \mathrm{H} 2$ and $98 \%$ O2) during cardiopulmonary resuscitation in a rat model of cardiac arrest, with treated animals having improved survival and fewer neurological deficits compared with animals who underwent therapeutic hypothermia (43).

The anti-inflammatory, antioxidant and beneficial effects of hydrogen have also been documented in a number of other acute oxidative stress settings, including a rat model of uncontrolled hemorrhagic shock, where hydrogen-rich saline $(>0.6 \mathrm{mM})$ had better activity when administered via the intravenous versus intraperitoneal route (44).

\section{Organ transplantation}

Preclinical data show that the antioxidant effects of hydrogen therapy are evident in organ transplantation models. In a rat model of renal transplant, treatment with hydrogen-enriched water versus regular water reduced chronic allograft nephropathy and improved overall post-transplant survival; these effects appeared to be secondary to reductions in oxidative stress-induced tissue damage (45). Hydrogen-enriched water $(0.5$ or $0.6 \mathrm{mM})$ has also been shown to protect rat cardiac allografts from oxidative injury and inflammation-associated deterioration, with significant reductions in markers of oxidative injury and of inflammation $(p<0.05)$, thereby increasing allograft survival (46). Moreover, hydrogenenriched water $(0.55-0.65 \mathrm{mM})$ inhibited intimal hyperplasia and reduced oxidative damage in arterialized vein grafts in rats (47). Inhaled hydrogen (2\%) appears to have beneficial effects in models of lung and intestinal transplantation, including improved spontaneous muscle contractile activity, reduced expression of inflammatory mediators and oxidative injury, prevention of IRI and improved graft survival $(48,49)$.

\section{Inflammation and ulceration}

The anti-inflammatory effect of hydrogen is related to the inhibition of the production of ROS and the release of pro-inflammatory cytokines.

In a rat model of ulcerative colitis (UC), intraperitoneal injection of hydrogen-enriched saline $(0.6 \mathrm{mM})$ was shown to reduce weight loss and diarrhea, as well as alleviate colonic mucosal damage (50). Furthermore, hydrogen-enriched saline was also associated with inhibition of the increased expression of vascular endothelial growth factor seen in this UC model.

In another study, four patients with acute erythematous skin disease were found to experience rapid and significant improvements in symptoms after intravenous treatment with $500 \mathrm{~mL}$ of hydrogen-enriched water, with no subsequent recurrence (51).

Hydrogen enriched water (600 mL/ day: 0.8-1.3 ppm) improved pressure ulcers in 12/22 (55\%) of hospitalized elderly patients. The responders had a significant reduction in hospital stay (113.3 days vs 155.4 days; $\mathrm{p}<0.05)$ and in the number of pressure ulcers $(28.1 \%$ reduction) compared with the subgroup of patients for whom hydrogen treatment was not effective. Both groups of hydrogen-treated patients showed a significant reduction in wound size from baseline, which was greater in the effective group. The results of parallel in vitro studies led study investigators to suggest that the beneficial effects of hydrogen therapy were secondary to type-I collagen construction in dermal fibroblasts and/ or promoted mitochondrial reducing ability and ROS repression in epidermal keratinocytes (52).

Twenty patients with rheumatoid arthritis (RA), drank 
$530 \mathrm{~mL}$ of hydrogen-rich water (4-5 ppm) every day for 4 weeks in addition to regular RA medication. This treatment period was followed by a 4 -week washout then another 4 weeks of treatment (53). There was a mean decrease of $14.3 \%$ from baseline in 8-hydroxyguanine (8-OHdG is a standard biomarker for oxidative stress) during the first 4 weeks of treatment $(p<0.01)$ and after the second period of hydrogen-enriched water therapy the reduction in 8 OHdG levels was $15.1 \%$ ( $p<0.01$ vs baseline). Disease activity scores significantly decreased in $18 / 20$ patients during the first 4 -weeks $(p<0.01)$ and remained lower than baseline levels at the end of the 4 -week washout phase. At study end, 16 patients had improved disease activity scores and nine patients achieved remission. Four patients had not received any previous RA treatment and all achieved remission during the 12-week study. Apart from increased urinary frequency due to an increased fluid intake, no adverse effects of hydrogen were observed, suggesting that hydrogen water might be a useful addition to conventional therapy in patients with RA $(53,54)$.

\section{Metabolic disorders}

Oxidative stress plays a role in the development of type 2 diabetes mellitus, suggested by accumulation of molecular hydrogen in the liver with glycogen after oral administration of hydrogen water in rats (55), making this another possible target for hydrogen therapy. The consumption of hydrogen-enriched water reduced oxidative stress in the liver, improved fatty liver in mice with diabetes and in those with diet-induced obesity reduced plasma glucose and triglyceride levels, attenuated body weight gain, and stimulation of energy metabolism (55).

Patients with type 2 diabetes or impaired glucose tolerance were included an 8-week randomized, doubleblind, crossover study of hydrogen-enriched water $(1.2 \pm$ $0.1 \mathrm{mg} / \mathrm{L}$ ) (56). Hydrogen was associated with significant reductions in levels of modified LDL cholesterol, small dense LDL and urinary 8-isoprostanes, plus smaller reductions in serum levels of oxidized LDL and free fatty acids and increased plasma levels of adiponectin and extracellular-superoxide dismutase (56).

Hydrogen-enriched water or saline $(>0.6 \mathrm{mM})$ has been shown to contribute to atherosclerosis prevention in apolipoprotein-E knockout mice $(57,58)$ and improved plaque stability (59). Additionally, ad libitum access to hydrogen-enriched water $(>0.8 \mathrm{mM})$ ameliorated pulmonary arterial hypertension in a rat model (60), and intraperitoneal administration of hydrogenenriched saline $(>0.6 \mathrm{mM})$ reduced oxidative stress and attenuated left ventricular hypertrophy in spontaneously hypertensive rats (61).

Three clinical trials have assessed the effects of hydrogen treatment in the metabolic syndrome setting. After 10 weeks of hydrogen water (0.2-
$0.25 \mathrm{mM}$ ), significant reductions from baseline were seen in serum levels of total and low-density lipoprotein (LDL) cholesterol, apolipoproteins B and $\mathrm{E}$, and malondialdehyde (MDA; an indicator of lipid peroxidation). Improvements were also seen in oxidation of LDL and high-density lipoprotein (HDL), and in LDL-mediated inflammation (62). In a similar group of potential metabolic syndrome patients, consumption of $1.5-2 \mathrm{~L}$ of hydrogen-enriched water $(0.55-0.65 \mathrm{mM})$ per day for 8 weeks increased HDL cholesterol levels and decreased the total cholesterol/HDL cholesterol ratio compared with baseline (63).

\section{Neurodegenerative diseases}

Evidence of increased levels of ROS have been found in post-mortem samples of brain tissue samples from patients with neurodegenerative diseases (3). There are a number of factors that make the brain particularly susceptible to oxidative stress, including its high metabolic rate and reduced capacity for cellular regeneration compared with other organs $(3,64)$.

Consumption of hydrogen-enriched water $(>0.6$ $\mathrm{mM}$ ) ad libitum suppressed increases in oxidative stress markers and improved the proliferation of neural progenitor cells in a mouse chronic physical restraint model (65). Hydrogen-enriched saline reduced oxidative stress, inflammatory responses and amyloid-beta-induced astrocyte activation while improving learning and memory in a rat model of Alzheimer's disease (66).

In Parkinson's disease addition of hydrogen to drinking water $(>0.4 \mathrm{mM}$ or $0.08-1.5 \mathrm{ppm}$ ) prevented the development and progression of nigrostriatal degeneration and dopaminergic neuron loss $(67,68)$.

The efficacy of hydrogen-enriched water was assessed in a randomized, placebo-controlled, doubleblind, parallel clinical trial conducted as a pilot study in levodopa-treated patients with Parkinson's disease (69). Participants drank 1 L per day of hydrogenenriched water (prepared by dissolving $0.8 \mathrm{mM} \mathrm{H} 2$; $\mathrm{n}=9)$ or placebo water $(\mathrm{n}=8)$ for 48 weeks. Total Unified Parkinson's Disease Rating Scale (UPDRS) scores in the hydrogen-enriched water group improved during the study, whereas UPDRS scores in the placebo group worsened ( $p<0.05$ at 48 weeks). Hydrogen-enriched water was well tolerated during the study and no adverse events were reported.

\section{Spinal cord and brain injury}

Intraperitoneal administration of hydrogen-enriched saline $(0.6 \mathrm{mM})$ to rats at the time of spinal cord injury, and at 24 and 48 hours after injury was associated with a reduction in the number of apoptotic cells, decreased oxidative stress, increased release of brain-derived neurotrophic factor, and improved locomotor function (70). Similar beneficial effects were observed in a rat 
model of traumatic brain injury (TBI) after treatment with $2 \%$ inhaled hydrogen in air (71). Hydrogen was administered from 5 minutes to 5 hours after TBI, and significantly attenuated TBI-induced increases in bloodbrain barrier permeability, brain edema, lesion volume and neurological dysfunction. These effects were thought to occur via increases in endogenous antioxidant enzyme activities (71). Reduction in brain edema and protection from the neurodegenerative changes associated with TBI has also been documented when hydrogen is added to drinking water $(>1.6 \mathrm{mM})$, starting 24 hours before TBI. In particular, expression of genes relating to oxidation, neuroinflammation and carbohydrate metabolism was effectively reversed by hydrogen water (72).

\section{Cancer}

\section{Anti-cancer effects}

The production of ROS by human cancer cells is higher than that for normal cell lines (73). In addition, elevated levels of ROS appear to play a role in the signaling cascades that trigger and maintain the oncogenic phenotype of cancer cells (74), and may contribute to DNA damage, and cell proliferation, migration and survival (75). Therefore, the possible anticancer effects of hydrogen, alone or in combination with traditional therapy, are the subject of growing research (76).

In an in vitro study, hydrogen-enriched water (1.0-1.3 ppm) supplemented with platinum nanocolloid showed rapid antioxidant effects and preferentially inhibited clonal growth of human tongue carcinoma cells compared with normal cells (77). Hydrogen-enriched water has also been shown to increase the inhibitory effects of the chemotherapy agent fluorouracil on colon cancer cells (76). In a mouse model of colon cancer, hydrogenenriched water $(\approx 0.8 \mathrm{mM})$ significantly increased survival compared with placebo water treatment, and survival was increased to an even greater extent when hydrogenenriched water was combined with fluouracil (76).

\section{Radiation toxicities}

Radiotherapy is associated with radiolysis of $\mathrm{H} 2 \mathrm{O}$ to $\bullet \mathrm{OH}$, which contributes to a range of adverse effects (33). The results of one preclinical study showed that hydrogen-enriched saline $(>0.6 \mathrm{mM})$ protects cells against radiation-induced toxicity and ameliorates intestinal injury caused by radiation (78). Another showed that hydrogen-enriched saline $(>0.6 \mathrm{mM})$ markedly reduced ROS levels after radiation and protected against radiation-induced immune dysfunction (79), while a third indicated that treatment with hydrogen-enriched saline $(0.6 \mathrm{mM})$ before ionizing radiation could provide partial protection of both spermatogenesis and hematopoiesis (80). Hydrogen has been proposed as a possible preventive strategy for radiation pneumonitis because of its ability to quickly scavenge free radicals produced by irradiation of the lungs (81).

The effects of drinking hydrogen-enriched water were investigated in a randomized, placebo-controlled study of patients receiving radiotherapy for liver tumors (82). Treatment with 1.5-2L hydrogen-enriched $(0.55-0.65 \mathrm{mM})$ or placebo water was started on the first day of radiation treatment and continued for 6 weeks. Drinking hydrogenenriched water prevented the marked increase in total serum hydroperoxide associated with radiotherapy, and attenuated the radiation-related reduction in serum antioxidant activity. Patients in the hydrogen-enriched water group reported significantly less loss of appetite and fewer taste disorders compared with those receiving placebo. Importantly, hydrogen-enriched water had no effect on the efficacy of radiotherapy (82).

Side effects of cisplatin

Based on the results of an in vivo study, hydrogen also has the potential to alleviate chemotherapy-associated toxicity. Two administration routes (oral hydrogenenriched drinking water $[0.8 \mathrm{mM}]$ and inhaled hydrogen [1\%]) were assessed in mice receiving cisplatin (83). Both forms of hydrogen improved survival, reduced body weight loss and ameliorated cisplatin-induced nephrotoxicity compared with control, although the effects of drinking hydrogen-enriched water appeared to be less than those of inhaled hydrogen with respect to prevention of severe nephrotoxicity. The anti-tumor efficacy of cisplatin was unaffected by concomitant administration of hydrogen by either route (83).

\section{Other medical applications}

\section{Dermatomyositis and mitochondrial disease}

The effects of drinking hydrogen-enriched water (0.5 ppm) were assessed in patients with muscle diseases (84). An open-label study was conducted in 14 patients who drank $1 \mathrm{~L} /$ day of hydrogen-enriched water for 12 weeks. Patients with mitochondrial myopathies (MM) and patients with progressive muscular dystrophy showed decreases in the lactate-to-pyruvate ratio $(\mathrm{p}<0.05)$, and those with polymyositis / dermatomyositis had a decrease in serum matrix metalloproteinase- 3 and triglyceride levels (84). Subsequently, a randomized, double-blind, placebo-controlled, crossover study in 22 patients with dermatomyositis $(n=10)$ or MM $(n=12)$ investigated the effects of drinking $0.5 \mathrm{~L} /$ day of hydrogen-enriched water for 8 weeks. Treatment was associated with significant improvement in serum lactate in MM patients, but there were no objective changes in clinical symptoms in this or the open-label study (84). Some patients did report subjective improvements in fatigue, diarrhea and 
myalgia, but these were not consistent or statistically significant suggesting that the hydrogen dosage used may have been insufficient. It was suggested that $>1 \mathrm{~L} /$ day of hydrogen-enriched water may be required to achieve beneficial effects (84).

\section{Hemodialysis}

The effects of using hydrogen-enriched water as a hemodialysis solution have been investigated in two clinical trials. In the first, regular hemodialysis patients received one month of treatment using a standard solution followed by another month of treatment using hydrogen-enriched water (99 parts per billion [ppb]) as the dialysis solution (85). Hemodialysis with both the standard and hydrogen-enriched water solutions was effective at reducing blood pressure. Compared with standard solution, hydrogen-enriched water was associated with preserved cellular viability of polymorphonuclear leukocytes, resulting in better ability to generate superoxide anions. Use of hydrogenenriched water during hemodialysis was feasible and well tolerated, with no symptomatic adverse events observed during the study period (85). The second clinical trial included a larger number of patients $(n=21)$ dialyzed with hydrogen-enriched solution (average H2 levels ranged from $48 \mathrm{ppb}$ to $210 \mathrm{ppb}$ ) over a longer period of time (6 months) (24). Once again, hemodialysis with hydrogen water had a good tolerability profile and was feasible, and significant reductions in blood pressure were achieved after each hemodialysis session; these were greater when hemodialysis was performed with hydrogen versus standard solution. Significant decreases were also observed in the oxidant markers plasma myeloperoxidase and monocyte chemoattractant protein-1 (24). These results suggest that use of hydrogenenriched solutions during hemodialysis has the potential to ameliorate inflammatory reactions and improve blood pressure control.

\section{Liver disease}

Lipid peroxidation plays an important role in the pathology of viral hepatitis and has been suggested as a major cause of liver injury (86). The addition of hydrogenenriched water $(0.55-0.65 \mathrm{mM}$; three times daily) to standard treatment for 6 weeks in patients with chronic hepatitis B was associated with significant improvements in a number of oxidative stress parameters, including superoxide dismutase, glutathione $S$ transferase, xanthine oxidase and malondialdehyde compared with both baseline $(\mathrm{p}<0.05)$ and the standard treatment only group $(p<0.01)(87)$. There were trends towards improvements in liver function and reductions in hepatitis B virus DNA load in the hydrogen treatment versus routine treatment group, but these did not reach statistical significance (87).

\section{Respiratory system}

The role of oxidative stress in the development of acute lung injury is well established (33). In animal models, hydrogen-enriched saline and inhaled hydrogen $(2 \%)$ reduced apoptosis, neutrophil infiltration, lipid membrane peroxidation, nuclear factor kappa B activation and proinflammatory cytokine levels (88-90).

\section{Ocular effects}

There are a number of ocular diseases that have been shown to benefit from hydrogen treatment in preclinical studies. Hydrogen-enriched saline has been shown to have antioxidant effects and to protect against blue lightinduced retinal damage (91) and retinopathy associated with hyperoxia or diabetes $(92,93)$, and to prevent selenite-induced cataract (94).

\section{Conclusion}

Hydrogen is an effective antioxidant, antiinflammatory and cytoprotective agent that selectively scavenges potent oxidants such as $\bullet \mathrm{OH}$ and peroxynitrite in cells without affecting redox homeostasis. Key features of hydrogen as a therapeutic agent include selective reaction with highly reactive ROS, rapid diffusion into cells and onset of action, inert characteristics at body temperature in mammalian cells, an almost adverse event-free tolerability profile, the ability to administer therapy in a variety of ways to best suit the patient or indication being treated, and low cost. The non-specific nature of the effects of hydrogen mean that it has therapeutic potential across a wide range of medical applications, as has been shown by a good volume of preclinical data and a growing body of clinical evidence.

Accumulating data also show the potential for hydrogen as an anti-ageing solution and in wellness applications, particularly sports and injury. It is possible that drinking hydrogen-enriched water may become a novel and beneficial new rehydration strategy for athletes. Data from future clinical studies will help to better define the role of hydrogen in medical applications, exercise physiology and wellness strategies. Hydrogen has the potential to become a hugely important agent in many aspects of human health, particularly in reducing the effects of ageing.

Disclosure: Marc Lemaire and Françoise Barbier are employees of Air Liquide.

Acknowledgements: The authors would like to thank Marie Cheeseman and Nicola Ryan who provided medical writing assistance on behalf of Springer Healthcare Communications. This assistance was funded by Air Liquide.. 


\section{References}

1. Bavykin DV. A. Zuttel, A. Borgschulte, L. Schlapbach (eds.): Hydrogen as a Future Energy Carrier. J Appl Electrochem 2008;38:1483-1483.

2. Ohta S. Molecular hydrogen as a preventive and therapeutic medical gas: initiation, development and potential of hydrogen medicine. Pharmacol Ther 144:1-11.

3. Andersen JK. Oxidative stress in neurodegeneration: cause or consequence? Nat Med 2004;10 Suppl:S18-25.

4. El Assar M, Angulo J, Rodriguez-Manas L. Oxidative stress and vascular inflammation in aging. Free radical biology \& medicine2013;65:380-401.

5. Kim YW, Byzova TV. Oxidative stress in angiogenesis and vascular disease. Blood 2014;123:625-631.

6. Reuter S, Gupta SC, Chaturvedi MM, Aggarwal BB. Oxidative stress, inflammation, and cancer: how are they linked? Free radical biology \& medicine 2010;49:1603-1616.

7. Vaziri ND, Rodriguez-Iturbe B. Mechanisms of disease: oxidative stress and inflammation in the pathogenesis of hypertension. Nature clinical practice Nephrology 2006;2:582-593.

8. Steinhubl SR. Why have antioxidants failed in clinical trials? Am J Cardiol 2008;101:14D-19D.

9. Ohta S. Molecular hydrogen is a novel antioxidant to efficiently reduce oxidative stress with potential for the improvement of mitochondrial diseases. Biochim Biophys Acta 2012;1820:586-594

10. Ohta S, Nakao A, Ohno K. The 2011 Medical Molecular Hydrogen Symposium: An inaugural symposium of the journal Medical Gas Research. Med Gas Res 2011;1:10.

11. Ohsawa I, Ishikawa M, Takahashi $\mathrm{K}$ et al. Hydrogen acts as a therapeutic antioxidant by selectively reducing cytotoxic oxygen radicals. Nat Med 2007;13:688-694

12. Ghanizadeh A, Berk M. Molecular hydrogen: an overview of its neurobiological effects and therapeutic potential for bipolar disorder and schizophrenia. Med Gas Res 2013;3:11.

13. Rosen GM, Pou S, Ramos CL, Cohen MS, Britigan BE. Free radicals and phagocytic cells. Faseb J 1995;9:200-209.

14. Itoh $\mathrm{T}$, Hamada $\mathrm{N}$, Terazawa $\mathrm{R}$ et al. Molecular hydrogen inhibits lipopolysaccharide/interferon gamma-induced nitric oxide production through modulation of signal transduction in macrophages. Biochem Biophys Res Commun 2011;411:143-149.

15. Cai J, Kang Z, Liu WW et al. Hydrogen therapy reduces apoptosis in neonatal hypoxia-ischemia rat model. Neurosci Lett 2008;441:167-172.

16. Dixon BJ, Tang J, Zhang JH. The evolution of molecular hydrogen: a noteworthy potential therapy with clinical significance. Med Gas Res 2013;3:10

17. Kurokawa R, Seo T, Sato B, Hirano S, Sato F. Convenient methods for ingestion of molecular hydrogen: drinking, injection, and inhalation. Med Gas Res 2015;5:13

18. Young CL, editor. Hydrogen and Deuterium: Pergamon Press; 1981.

19. Barouki R. ) [Ageing free radicals and cellular stress]. Med Sci (Paris) 2006;22:266-272

20. Huang CS, Kawamura T, Toyoda Y, Nakao A. Recent advances in hydrogen research as a therapeutic medical gas. Free Radic Res 2010;44:971-982.

21. Okouchi S, Takezai D, Ohnami H, Agishi Y, Kanroji Y, Ikeda S. Electrolyzedreduced water as artificial hot spring water. Journal of the Balneological Society of Japan 2003;53:1-9.

22. Okouchi S, Ohnami H, Shoji M et a. Effect of electrolyzed-reduced water as artificial hot spring on human skin and hair. Journal of the Balneological Society of Japan 2005;55:55-63.

23. Meffert H, Diezel W, Sonnichsen N. Stable lipid peroxidation products in human skin: detection, ultraviolet light-induced increase, pathogenic importance. Experientia 1976;32:1397-1398.

24. Nakayama M, Nakano H, Hamada H, Itami N, Nakazawa R, Ito S. A novel bioactive haemodialysis system using dissolved dihydrogen (H2) produced by water electrolysis: a clinical trial. Nephrol Dial Transplant 2010;25:30263033.

25. Shirahata S, Kabayama S, Nakano M et al. Electrolyzed-reduced water scavenges active oxygen species and protects DNA from oxidative damage. Biochem Biophys Res Commun1997;234:269-274.

26. Xue J, Shang G, Tanaka Y et al. Dose-dependent inhibition of gastric injury by hydrogen in alkaline electrolyzed drinking water. BMC Complement Altern Med 2014;14:81.

27. Powers SK, Jackson MJ. Exercise-induced oxidative stress: cellular mechanisms and impact on muscle force production. Physiol Rev 2008;88:1243-1276.

28. Ostojic SM. Serum alkalinization and hydrogen-rich water in healthy men. Mayo Clin Proc 2012;87:501-502.

29. Ostojic SM, Stojanovic MD, Calleja-Gonzalez J, Obrenovic MD, Veljovic D, Medjedovic B. Drink with alkaline NORP improve exercise performance in physically active men and women. Serb J Sports Sci 2011;5:83-89.

30. Aoki K, Nakao A, Adachi T, Matsui Y, Miyakawa S. Pilot study: Effects of drinking hydrogen-rich water on muscle fatigue caused by acute exercise in elite athletes. Med Gas Res 2012:2:12.

31. Ostojic SM, Stojanovic MD, Hoffman JR. Effectiveness of molecular hydrogen in the management of musculotendinous injuries. Med Sci Sports Exerc 2014;46:S156-S157.

32. Sakai T, Sato B, Hara K et al. Consumption of water containing over $3.5 \mathrm{mg}$ of dissolved hydrogen could improve vascular endothelial function. Vasc Health Risk Manag 2014;10:591-597.

33. Shen M, Zhang H, Yu C, Wang F, Sun X. A review of experimental studies of hydrogen as a new therapeutic agent in emergency and critical care medicine. Med Gas Res 2014;4:17.

34. Hayashida K, Sano M, Ohsawa I et al. Inhalation of hydrogen gas reduces infarct size in the rat model of myocardial ischemia-reperfusion injury. Biochem Biophys Res Commun 2008;373:30-35.

35. Sun Q, Kang Z, Cai J et al. Hydrogen-rich saline protects myocardium against ischemia/reperfusion injury in rats. Exp Biol Med 2009:234:1212-1219.

36. Zhang Y, Sun Q, He B, Xiao J, Wang Z, Sun X. Anti-inflammatory effect of hydrogen-rich saline in a rat model of regional myocardial ischemia and reperfusion. International journal of cardiology 2011:148:91-95.

37. Shinbo T, Kokubo K, Sato Y et al. Breathing nitric oxide plus hydrogen gas reduces ischemia-reperfusion injury and nitrotyrosine production in murine heart. American journal of physiology Heart and circulatory physiology 2013;305:H542-550

38. Fukuda K, Asoh S, Ishikawa M, Yamamoto Y, Ohsawa I, Ohta S. Inhalation of hydrogen gas suppresses hepatic injury caused by ischemia/reperfusion through reducing oxidative stress. Biochem Biophys Res Commun 2007:361:670-674

39. Liu Y, Yang L, Tao K et al. Protective effects of hydrogen enriched saline on liver ischemia reperfusion injury by reducing oxidative stress and HMGB1 release. BMC gastroenterology 2014;14:12.

40. Wang F, Yu G, Liu SY et al. Hydrogen-rich saline protects against renal ischemia/reperfusion injury in rats. J Surg Res 2011;167:e339-344.

41. Ono $\mathrm{H}$, Nishijima $\mathrm{Y}$, Adachi $\mathrm{N}$ et al. Improved brain MRI indices in the acute brain stem infarct sites treated with hydroxyl radical scavengers, Edaravone and hydrogen, as compared to Edaravone alone. A non-controlled study. Med Gas Res 2011:1:12.

42. Huang G, Zhou J, Zhan $\mathrm{W}$ et al. The neuroprotective effects of intraperitoneal injection of hydrogen in rabbits with cardiac arrest. Resuscitation 2013;84:690695.

43. Hayashida K, Sano M, Kamimura N et al. H(2) gas improves functional outcome after cardiac arrest to an extent comparable to therapeutic hypothermia in a rat model. Journal of the American Heart Association 2012;1:e03459.

44. Du Z, Jia H, Liu J, Zhao X, Wang Y, Sun X. Protective effects of hydrogenrich saline in uncontrolled hemorrhagic shock. Experimental and therapeutic medicine 2014;7:1253-1258.

45. Cardinal JS, Zhan J, Wang Y et al. Oral hydrogen water prevents chronic allograft nephropathy in rats. Kidney Int 2010;77:101-109.

46. Noda K, Tanaka $\mathrm{Y}$, Shigemura $\mathrm{N}$ et al. Hydrogen-supplemented drinking water protects cardiac allografts from inflammation-associated deterioration. Transpl Int 2012;25:1213-1222.

47. Sun $\mathrm{Q}$, Kawamura $\mathrm{T}$, Masutani $\mathrm{K}$ et al. Oral intake of hydrogen-rich water inhibits intimal hyperplasia in arterialized vein grafts in rats. Cardiovasc Res 2012;94:144-153.

48. Buchholz BM, Kaczorowski DJ, Sugimoto R et al. Hydrogen inhalation ameliorates oxidative stress in transplantation induced intestinal graft injury. Am J Transplant 2008;8:2015-2024.

49. Kawamura T, Huang CS, Tochigi $\mathrm{N}$ et al. Inhaled hydrogen gas therapy for prevention of lung transplant-induced ischemia/reperfusion injury in rats. Transplantation 2010;90:1344-1351.

50. He J, Xiong S, Zhang J et al. Protective effects of hydrogen-rich saline on ulcerative colitis rat model. J Surg Res 2013;185:174-181.

51. Ono H, Nishijima $\mathrm{Y}$, Adachi $\mathrm{N}$ et al. Hydrogen $(\mathrm{H} 2)$ treatment for acute erythymatous skin diseases. A report of 4 patients with safety data and a non-controlled feasibility study with $\mathrm{H} 2$ concentration measurement on two volunteers. Med Gas Res 2012;2:14.

52. Li Q, Kato S, Matsuoka D, Tanaka H, Miwa N. Hydrogen water intake via tube-feeding for patients with pressure ulcer and its reconstructive effects on normal human skin cells in vitro. Med Gas Res2013;3:20.

53. Ishibashi $\mathrm{T}$, Sato $\mathrm{B}$, Rikitake $\mathrm{M}$ et al. Consumption of water containing a high concentration of molecular hydrogen reduces oxidative stress and disease activity in patients with rheumatoid arthritis: an open-label pilot study. Med Gas Res 2012;2:27.

54. Ishibashi T. Molecular hydrogen: new antioxidant and anti-inflammatory therapy for rheumatoid arthritis and related diseases. Curr Pharm Des 2013;19:6375-6381.

55. Kamimura N, Nishimaki K, Ohsawa I, Ohta S. Molecular hydrogen improves obesity and diabetes by inducing hepatic FGF21 and stimulating energy metabolism in db/db mice. Obesity (Silver Spring) 2011;19:1396-1403.

56. Kajiyama S, Hasegawa G, Asano M et al. Supplementation of hydrogen-rich water improves lipid and glucose metabolism in patients with type 2 diabetes or impaired glucose tolerance. Nutr Res 2008;28:137-143

57. Ohsawa I, Nishimaki K, Yamagata K, Ishikawa M, Ohta S. Consumption of 
hydrogen water prevents atherosclerosis in apolipoprotein E knockout mice. Biochem Biophys Res Commun 2008;377:1195-1198.

58. Song G, Tian H, Qin S et al. Hydrogen decreases athero-susceptibility in apolipoprotein B-containing lipoproteins and aorta of apolipoprotein E knockout mice. Atherosclerosis 2012;221:55-65.

59. Song G, Zong C, Zhang Z et al. Molecular Hydrogen stabilizes atherosclerotic plaque in low-density lipoprotein receptor knockout mice. Free radical biology \& medicine, 2015.

60. Kishimoto $\mathrm{Y}$, Kato $\mathrm{T}$, Ito $\mathrm{M}$ et al. Hydrogen ameliorates pulmonary hypertension in rats by anti-inflammatory and antioxidant effects. J Thorac Cardiovasc Surg, 2015.

61. Yu YS, Zheng H. Chronic hydrogen-rich saline treatment reduces oxidative stress and attenuates left ventricular hypertrophy in spontaneous hypertensive rats. Mol Cell Biochem 2012;365:233-242.

62. Song G, Li M, Sang H et al. Hydrogen-rich water decreases serum LDLcholesterol levels and improves HDL function in patients with potential metabolic syndrome. J Lipid Res 2013;54:1884-1893.

63. Nakao A, Toyoda Y, Sharma P, Evans M, Guthrie N. Effectiveness of hydrogen rich water on antioxidant status of subjects with potential metabolic syndrome-an open label pilot study. J Clin Biochem Nutr 2010;46:140-149.

64. Federico A, Cardaioli E, Da Pozzo P, Formichi P, Gallus GN, Radi E. Mitochondria, oxidative stress and neurodegeneration. J Neurol Sci 2012;322:254-262.

65. Nagata K, Nakashima-Kamimura N, Mikami T, Ohsawa I, Ohta S. Consumption of molecular hydrogen prevents the stress-induced impairments in hippocampus-dependent learning tasks during chronic physical restraint in mice. Neuropsychopharmacology 2009;34:501-508.

66. Li J, Wang C, Zhang JH, Cai JM, Cao YP, Sun XJ. Hydrogen-rich saline improves memory function in a rat model of amyloid-beta-induced Alzheimer's disease by reduction of oxidative stress. Brain Res 2010;1328:152161.

67. Fu Y, Ito M, Fujita $\mathrm{Y}$ et al. Molecular hydrogen is protective against 6-hydroxydopamine-induced nigrostriatal degeneration in a rat model of Parkinson's disease. Neurosci Lett 2009:453:81-85.

68. Fujita K, Seike T, Yutsudo $\mathrm{N}$ et al. Hydrogen in drinking water reduces dopaminergic neuronal loss in the 1-methyl-4-phenyl-1,2,3,6tetrahydropyridine mouse model of Parkinson's disease. PLoS One 2009; $4: \mathrm{e} 7247$.

69. Yoritaka A, Takanashi M, Hirayama M, Nakahara T, Ohta S, Hattori N. Pilot study of H(2) therapy in Parkinson's disease: a randomized double-blind placebo-controlled trial. Mov Disord 2013;28:836-839.

70. Chen C, Chen Q, Mao Y et al. Hydrogen-rich saline protects against spinal cord injury in rats. Neurochemical research 2010;35:1111-1118.

71. Ji X, Liu W, Xie K et al. Beneficial effects of hydrogen gas in a rat model of traumatic brain injury via reducing oxidative stress. Brain Res 2010;1354:196205.

72. Dohi K, Kraemer BC, Erickson MA et al. Molecular hydrogen in drinking water protects against neurodegenerative changes induced by traumatic brain injury. PLoS One2014;9:e108034.

73. Szatrowski TP, Nathan CF. Production of large amounts of hydrogen peroxide by human tumor cells. Cancer research 1991;51:794-798.

74. Behrend L, Henderson G, Zwacka RM. Reactive oxygen species in oncogenic transformation. Biochemical Society transactions 2003;31:1441-1444.

75. Storz P. Reactive oxygen species in tumor progression. Frontiers in bioscience : a journal and virtual library 2005;10:1881-1896.
76. Runtuwene J, Amitani H, Amitani M, Asakawa A, Cheng KC, Inui A. Hydrogen-water enhances 5-fluorouracil-induced inhibition of colon cancer. PeerJ 2015:3:e859.

77. Saitoh Y, Yoshimura Y, Nakano K, Miwa N. Platinum nanocolloidsupplemented hydrogendissolved water inhibits growth of human tongue carcinoma cells preferentially over normal cells. Exp Oncol 2009;31:156-162.

78. Qian L, Cao F, Cui J et al. Radioprotective effect of hydrogen in cultured cells and mice. Free Radic Res 2010;44:275-282.

79. Zhao S, Yang Y, Liu W et al. Protective effect of hydrogen-rich saline against radiation-induced immune dysfunction. J Cell Mol Med 2014;18:938-946.

80. Chuai Y, Shen J, Qian L et al. Hydrogen-rich saline protects spermatogenesis and hematopoiesis in irradiated BALB/c mice. Med Sci Monit 2012;18:BR8994.

81. Chuai $\mathrm{Y}, \mathrm{Zhao} \mathrm{L}, \mathrm{Ni}$ J et al. A possible prevention strategy of radiation pneumonitis: combine radiotherapy with aerosol inhalation of hydrogen-rich solution. Med Sci Monit 2011;17:HY1-4.

82. Kang KM, Kang YN, Choi IB et al. Effects of drinking hydrogen-rich water on the quality of life of patients treated with radiotherapy for liver tumors. Med Gas Res 2011;1:11.

83. Nakashima-Kamimura N, Mori T, Ohsawa I, Asoh S, Ohta S. Molecular hydrogen alleviates nephrotoxicity induced by an anti-cancer drug cisplatin without compromising anti-tumor activity in mice. Cancer Chemother Pharmacol 2009;64:753-761.

84. Ito M, Ibi T, Sahashi K, Ichihara M, Ohno K. Open-label trial and randomized, double-blind, placebo-controlled, crossover trial of hydrogen-enriched water for mitochondrial and inflammatory myopathies. Med Gas Res 2011;1:24.

85. Nakayama M, Kabayama S, Nakano H et al. Biological effects of electrolyzed water in hemodialysis. Nephron Clin Pract 2009;112:c9-15.

86. Muriel P. Role of free radicals in liver diseases. Hepatology international 2009;3:526-536.

87. Xia C, Liu W, Zeng D, Zhu L, Sun X. Effect of hydrogen-rich water on oxidative stress, liver function, and viral load in patients with chronic hepatitis B. Clin Transl Sci 6:372-375.

88. Mao YF, Zheng XF, Cai JM et al. Hydrogen-rich saline reduces lung injury induced by intestinal ischemia/reperfusion in rats. Biochem Biophys Res Commun2009;381:602-605.

89. Xiao M, Zhu T, Wang T, Wen FQ. Hydrogen-rich saline reduces airway remodeling via inactivation of NF-kappaB in a murine model of asthma. European review for medical and pharmacological sciences 2013;17:10331043.

90. Xie K, Yu Y, Huang Y et al. Molecular hydrogen ameliorates lipopolysaccharide-induced acute lung injury in mice through reducing inflammation and apoptosis. Shock 2012;37:548-555.

91. Feng $\mathrm{M}$, Wang XH, Yang XB, Xiao Q, Jiang FG. Protective effect of saturated hydrogen saline against blue light-induced retinal damage in rats. Int J Ophthalmol 2012;5:151-157.

92. Huang L, Zhao S, Zhang JH, Sun X. Hydrogen saline treatment attenuates hyperoxia-induced retinopathy by inhibition of oxidative stress and reduction of VEGF expression. Ophthalmic Res 2012;47:122-127.

93. Xiao X, Cai J, Xu J et al. Protective effects of hydrogen saline on diabetic retinopathy in a streptozotocin-induced diabetic rat model. J Ocul Pharmacol Ther 2012;28:76-82.

94. Yang CX, Yan H, Ding TB. Hydrogen saline prevents selenite-induced cataract in rats. Mol Vis 2013;19:1684-1693. 